\title{
Certain Generalized Prime elements
}

\author{
C. S. Manjarekar, A. N. Chavan
}

Shivaji University, Kolhapur, India

\begin{abstract}
In this paper we study different generalizations of prime elements and prove certain properties of these elements.

Keywords- Prime, primary elements,weakly prime elements,weakly primary elements, 2-absorbing, 2-potent elements

Math. Subject Classification Number:- 06F10, 06E20, $06 E 99$.
\end{abstract}

\section{INTRODUCTION}

A multiplicative lattice $\mathrm{L}$ is a complete lattice provided with commutative, associative and join distributive multiplication in which the largest element 1 acts as a multiplicative identity. An element $a \in L$ is called proper if a $<1$. A proper element $p$ of $L$ is said to be prime if $a b \leq p$ implies $a \leq p$ or $b \leq p$. If $a \in L, b \in L$,

$(a: b)$ is the join of all elements $c$ in L such that $c b \leq a$. A properelement $\mathrm{p}$ of $\mathrm{L}$ is said to be primary if $\mathrm{ab} \leq \mathrm{p}$ implies a $\leq \mathrm{p}$ or $b^{n} \leq \mathrm{p}$ for some positive integer $\mathrm{n}$. If $\mathrm{a} \in \mathrm{L}$, then $\sqrt{a}=$ $\vee\left\{\mathrm{x} \in L_{*} / x^{n} \leq \mathrm{a}, \mathrm{n} \in \mathrm{Z}+\right\}$. An element $\mathrm{a} \in \mathrm{L}$ is called a radical element ifa $=\sqrt{a}$. An element $a \in L$ is called compact if a $a \leq \mathrm{V}_{\alpha} b_{\alpha}$ implies $\mathrm{a} \leq b_{\alpha_{1}} \vee b_{\alpha_{2}} \vee \ldots \vee b_{\alpha_{n}}$ for some finite subset $\left\{\alpha_{1}, \alpha_{2} \ldots \alpha_{n}\right\}$. Throughout this paper, $\mathrm{L}$ denotes a compactly generated multiplicative lattice with 1 compact in which every finite product of compact element is compact. We shall denote by $L_{*}$, the set of compact elements of $\mathrm{L}$.

An element $i \in L$ is called 2-absorbing element if $a b c \leq i$ implies $a b \leq i$ or $b c \leq i$ or $c a \leq i$. A proper element $i \in L$ is called 2-absorbing primary if for all $\mathrm{a}, \mathrm{b}, \mathrm{c} \in \mathrm{L}, \mathrm{abc} \leq \mathrm{i}$ implies either $a b \leq \mathrm{i}$ or $\mathrm{bc} \leq \sqrt{i}$ or $\mathrm{ca} \leq \sqrt{i}$. This concept was defined by U.Tekir et.al. in [7]. It is observed that every prime element is 2-absorbing. An element $i \in \mathrm{L}$ is called semi-prime if $\mathrm{i}$ $=\sqrt{i}$. An element is i called 2-potent prime if $\mathrm{ab} \leq i^{2}$ implies $\mathrm{a} \leq \mathrm{i}$ or $\mathrm{b} \leq \mathrm{i}$. (See [6]). Every 2-absorbing element of $\mathrm{L}$ is a 2-absorbing primary element of $\mathrm{L}$. But the converse is not true. The element $\mathrm{q}=(12)$ is a 2-absorbing primary element of $\mathrm{L}$ but not 2-absorbing element of L. Also every primary element of $\mathrm{L}$ is a 2 absorbing primary element. But the converse is not true. The element $q=(6)$ is a 2 absorbing primary element of $\mathrm{L}$ but not a primary element of $\mathrm{L}$, since $\mathrm{L}$ is lattice of ideals of the ring. $\mathrm{R}=\langle\mathrm{Z},+,$.$\rangle .$ For all these definition one can refer [1],[4],[5].

\section{PRIME AND PRIMARY ABSORBING ELEMENTS}

The concept of primary 2-absorbing ideals was introduced by Tessema et.al. [5]. We generalize this concept for multiplicative lattices.

An element $i \in L$ is said to be weakly prime if $0 \neq a b \leq i$ implies $\mathrm{a} \leq \mathrm{i}$ or $\mathrm{b} \leq \mathrm{i}$.

It is easy to show that every prime element is 2- absorbing. Ex. The following table shows multiplication of elements in themultiplicative lattice $\mathrm{L}=0, \mathrm{p}, \mathrm{q}, 1$.
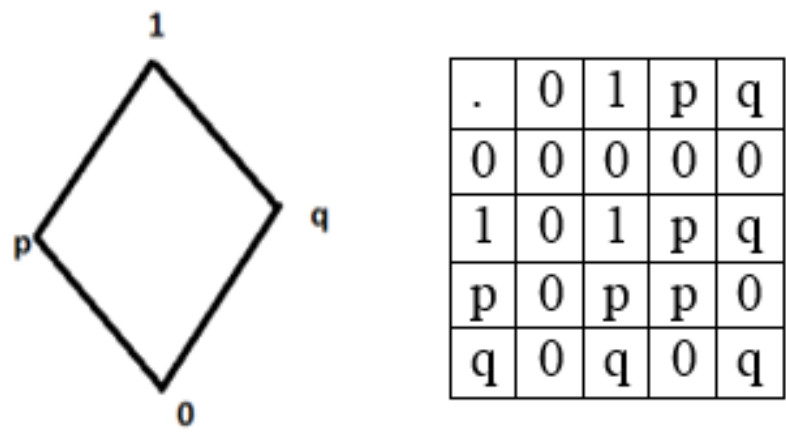

In the above diagram $0, \mathrm{p}, \mathrm{q}$ are 2 -absorbing.

The concept of 2-absorbing primary ideals is defined by A. Badawi,U. Tekir, E. Yetkin in [6]. The concept was generalized for multiplicative lattices by F. Calliap, E. Yetkin, and U. Tekir [8]. Weslightly modified this concept and defined primary 2-absorbing element.

Def.(2.1) An element $i \in \mathrm{L}$ is said to be weakly 2-absorbing if $\neq \neq a b c \leq i$ implies $a b \leq i$ or $b c \leq i$ or $c a \leq i$. (See [7]).

Def.(2.2) An element $\mathrm{i}$ of $\mathrm{L}$ is called primary 2-absorbing ifabc $\leq \mathrm{i}$ implies $\mathrm{ab} \leq \mathrm{i}$ or $\mathrm{bc} \leq \sqrt{i}$ or $\mathrm{ca} \leq \sqrt{i}$, for all $\mathrm{a}, \mathrm{b}, \mathrm{c} \in \mathrm{L}$.

Ex. Every 2- absorbing element of $L$ is primary 2absorbing.

We obtain now the relation between primary 2-absorbing element and 2-absorbing element.

Theorem (2.3) Ifi is semi-prime and primary 2-absorbing element of a lattice $\mathrm{L}$, then $\mathrm{i}$ is 2-absorbing.

Proof:- Suppose $\mathrm{i}$ is primary 2-absorbing. Let abc $\leq \mathrm{i}$. Then $\mathrm{ab} \leq \mathrm{i}$ or $\mathrm{bc} \leq \sqrt{i}$ or $\mathrm{ca} \leq \sqrt{i}$ where $\mathrm{i}=\sqrt{i}$. Therefore $\mathrm{i}$ is 2absorbing.

Theorem(2.4) If $\mathrm{i}$ is semi-prime and 2-potent prime element of Lthen $i$ is prime.

Proof:- Let $\mathrm{ab} \leq \mathrm{i}$. Then $(a b)^{2}=a^{2} b^{2} \leq i^{2}$. Then $a^{2} \leq \mathrm{i}$ or $b^{2} \leq \mathrm{i}$,since $\mathrm{i}$ is 2 -potent prime. This implies that $\mathrm{a} \leq \sqrt{i}$ or $\mathrm{b}$ 
$\leq \sqrt{i}$. Buti being semi-prime, $\mathrm{a} \leq \mathrm{i}$ or $\mathrm{b} \leq \mathrm{i}$. Hence iis a prime element.

we note that :A prime element is 2-potent prime.

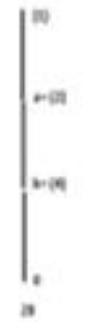

Consider the lattice $\mathrm{L}$ of ideals of ring $\mathrm{R}=\left\langle Z_{8},+, .>\right.$. Then the only ideals of $\mathrm{R}$ are principal ideals (0), (2), (4), (1). Clearly, L = (0), (2), (4), (1) is compactly generated multiplicative lattice. The element (4) $\in \mathrm{L}$ is not prime but it is 2-potent prime.

$\operatorname{Remark}(2.5)$ If $\mathrm{i}$ is semi-prime and primary then $\mathrm{i}$ is prime.

Now we establish the relation between 2-potent prime and primary element.

Theorem (2.6) Leti be a 2-potent prime. Then is almostprimary if and only if $\mathrm{i}$ is primary .

Proof:- Let $i$ be a primary element and $a b \leq i, a b \leq i^{2}$. Then a $\leq \mathrm{I}$ or $\mathrm{b} \leq \sqrt{i}$. So $\mathrm{i}$ is almost primary. Conversely, let $\mathrm{i}$ be an almostprimary element. Assume that $\mathrm{ab} \leq \mathrm{i}$. If $\mathrm{ab} \leq i^{2}$, then a $\leq \mathrm{i}$ orb $\leq \sqrt{i}$. Suppose $\mathrm{ab} \leq i^{2}$. Then $\mathrm{a} \leq \mathrm{i}$ or $\mathrm{b} \leq \mathrm{i} \leq \sqrt{i}$, since $\mathrm{i}$ is 2-potent prime. Therefore iis primary.

We obtain the relation between semiprime and 2-absorbing element.

Theorem(2.7) If $\mathrm{i}$ is semi-prime 2-potent prime element of L,then $\mathrm{i}$ is 2-absorbing.

(Proof:-) Let abc $\leq \mathrm{i}$. Then $(a b c)^{2}=(a b)^{2} c^{2} \leq i^{2}$. So $(a b)^{2} \leq \mathrm{i}$ orc ${ }^{2} \leq \mathrm{i}$, since $\mathrm{i}$ is 2-potent prime. As $\mathrm{i}$ is semi-prime, $\mathrm{ab} \leq \sqrt{i}$ $=\mathrm{i}$ or $\mathrm{c} \leq \sqrt{i}=\mathrm{i}$. Hence $\mathrm{ab} \leq \mathrm{i}$ or $\mathrm{bc} \leq \mathrm{i}$, ac $\leq \mathrm{i}$ and $\mathrm{i}$ is 2absorbing.

Remark (2.8) A 2-absorbing primary element need not be 2potent prime.

Ex.-Consider $\mathrm{L}$ as in example $Z_{30}$, the element $(6) \in \mathrm{L}$ is not2-potent prime.

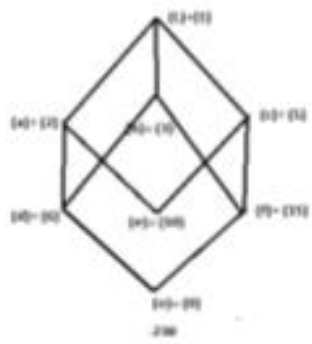

The next result gives the condition for a semi-prime element tobe almost primary.

Theorem (2.9) Leti be a semi-prime element of $\mathrm{L}$. Then iisalmost primary if $\mathrm{i}$ is weakly prime.

Proof:- Suppose $\mathrm{i}$ is weakly prime. Let $\mathrm{ab} \leq \mathrm{i}, \mathrm{ab} \not i^{2}$. Now $\mathrm{ab} \not i^{2}$ implies $\mathrm{ab} \neq 0$. Hence $\mathrm{a} \leq \mathrm{i}$ or $\mathrm{b} \leq \mathrm{i}=\sqrt{i}$.

Now we obtain a relation between primary element and weaklyprime element.

Theorem(2.10)Let $i$ be a semi-prime element. Then iis primaryif and only if $i$ is weakly prime.

Proof:- Assume that $\mathrm{i}$ is primary and $0 \neq \mathrm{ab} \leq \mathrm{i}$. Then $a^{n} \leq \mathrm{i}$ forsome $\mathrm{n} \in Z_{+}$or $\mathrm{b} \leq \mathrm{i}$. If $\mathrm{b} \leq \mathrm{i}$, we are done.If $\mathrm{an} \leq \mathrm{i}$, then $\mathrm{a}$ $\leq \sqrt{i}=\mathrm{i}$, since $\mathrm{i}$ is semi-prime. Hence is weaklyprime. Suppose iis weakly prime. Let $a b \leq i$. If $0 \neq a b$, then $a \leq i$ or $b$ $\leq \mathrm{i} \leq \sqrt{i}$ and $\mathrm{i}$ is primary. If $\mathrm{ab}=0 \leq \mathrm{i}, \mathrm{a}=0$ or $\mathrm{b}=0$ as $\mathrm{L}$ has no zero divisor. So $\mathrm{a} \leq \mathrm{i}$ or $\mathrm{b} \leq \sqrt{i}$ and $\mathrm{i}$ is primary.

Theorem (2.11) If an element $\mathrm{i}$ is both weakly primary andsemi-prime, then $\mathrm{i}$ is weakly prime.

Proof:- Let $0 \neq a b \leq \mathrm{i}$. Then $a^{n} \leq \mathrm{i}$ or $\mathrm{b} \leq \mathrm{i}$, since $\mathrm{i}$ weaklyprimary. If $\mathrm{b} \leq \mathrm{i}$, then an $\leq \mathrm{i}$ implies $\mathrm{a} \leq \mathrm{i}=\sqrt{i}$. Hence $\mathrm{i}$ is weaklyprime.

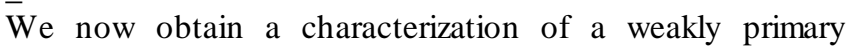
element.

Theorem (2.12) An element $i$ is weakly primary if and only if $(\mathrm{i}: \mathrm{x}) \leq \sqrt{i}$ or $(\mathrm{i}: \mathrm{x})=\left(i^{2}: \mathrm{x}\right)$ for all $\mathrm{x} \not \mathrm{i}$.

Proof:- Let $\mathrm{i}$ be weakly primary and $\mathrm{x} \not \mathrm{i}$. Since $i^{2} \leq \mathrm{i}$, we have $\left(i^{2}: \mathrm{x}\right) \leq(\mathrm{i}: \mathrm{x})$. Let $\mathrm{y} \leq(\mathrm{i}: \mathrm{x})$, then $\mathrm{yx} \leq \mathrm{i}$. If $\mathrm{yx}=0$, theny $\mathrm{x} \leq i^{2}$ which implies $\mathrm{y} \leq\left(i^{2}: \mathrm{x}\right)$. So in this case $(\mathrm{i}: \mathrm{x})$ $=\left(i^{2}: \mathrm{x}\right)$.

Suppose $y x \neq 0$. Then $y x \leq i, x \notin i$ and $i$ is weakly primary together imply $y^{n} \leq \mathrm{i}$ for some $\mathrm{n} \in \mathrm{Z}+$. Hence $\mathrm{y} \leq \sqrt{i}$ and(i : $\mathrm{x}) \leq \sqrt{i}$. Conversely suppose $(\mathrm{i}: \mathrm{x}) \leq \sqrt{i}$ or $(\mathrm{i}: \mathrm{x})=\left(i^{2}\right.$ : $\mathrm{x})$ whenever $\mathrm{x} \not \mathrm{i}$. Let $0 \neq \mathrm{ab} \leq \mathrm{i}$. If $\mathrm{b} \not \mathrm{i}$, we have nothing to prove. Otherwise $\mathrm{b} \leq \mathrm{i}$ and obviously $\mathrm{a} \leq(\mathrm{i}: \mathrm{b})$.

Case 1 If $\mathrm{a} \leq(\mathrm{i}: \mathrm{b}) \leq \sqrt{i}, \mathrm{i}$ is weakly primary.

Case 2) Suppose (i : b) $=\left(i^{2}:\right.$ b) and (i : b) $\$ \sqrt{i}$. In this case, there exists $\mathrm{z} \leq(\mathrm{i}: \mathrm{b})$ but $\mathrm{z} \notin \sqrt{i}$. Therefore $z^{n} \ddagger \mathrm{i}$ for all $\mathrm{n} \in \mathrm{Z}+$. 
Now $\mathrm{z} \leq(\mathrm{i}: \mathrm{b})$ implies $\mathrm{zb} \leq \mathrm{i}$ for $\mathrm{b} \not \mathrm{i}$. In particular, for $\mathrm{b}=$ $\mathrm{z}, \mathrm{z}^{2} \leq \mathrm{i}$, which is a contradiction. Hence the second case does notarises.

Theorem(2.13) Let $\mathrm{i}$ and $\mathrm{j}$ be distinct weakly prime elements of $\mathrm{L}$. Then $(i \wedge j)$ is weakly 2 - absorbing.

Proof:- Let $0 \neq a b c \leq(i \wedge j)$. Then $a b c \leq i$ and $a b c \leq j$. Since I and $\mathrm{j}$ are weakly prime elements, we have $a b \leq \mathrm{i}$ or $\mathrm{c} \leq \mathrm{i}$ and $\mathrm{ab} \leq \mathrm{j}$ or $\mathrm{c} \leq \mathrm{j}$.

Case 1) If $a b \leq i$ and $a b \leq j$ we have $a b \leq(i \wedge j)$.

Case 2) If $a b \leq i$ and $c \leq j$, then $a \leq i$ or $b \leq i$ and $c \leq j$, since $0 \neq$ $a b \leq i$ and $i$ is weakly prime. Thus $a c \leq i$, ac $\leq j$ or $b c \leq i$ andbc $\leq j$. This shows that $a c \leq(i \wedge j)$ or $b c \leq(i \wedge j)$.

Case 3) Let $c \leq i$ and $a b \leq j$. This case is similar to case (2).

Case 4) Suppose $c \leq i$ and $c \leq j$. In this case ac $\leq \mathrm{j}$, ac $\leq \mathrm{I}$ and thus ac $\leq(i \wedge j)$ together imply $(i \wedge j)$ is weakly 2 -absorbing.

Next we have a property of a weakly prime element.

Theorem (2.14) Leti be a weakly prime element of L. Then I is weakly 2-absorbing.

Proof:- Let $0 \neq a b c \leq i$. Then $a \leq i$ or $b c \leq i$. This again impliesa $\leq \mathrm{i}$ or $\mathrm{b} \leq \mathrm{i}$ or $\mathrm{c} \leq \mathrm{i}$. Hence $\mathrm{ab} \leq \mathrm{i}$ or $\mathrm{bc} \leq \mathrm{i}$ or $\mathrm{ac} \leq \mathrm{i}$ and $\mathrm{i}$ is weakly 2-absorbing.

\section{TWIN ZERO AND WEAKLY PRIME ELEMENTS}

The concept of a Twin zero of an ideal in a commutative rings withunity is introduced and studied in detail by A.Badawi et.al. [].

Wegeneralize this concept for multiplicative lattice and obtain some

results relating to this concept.

Definition 3.1) Let $L$ be a multiplicative lattice and $i \in$ $\mathrm{L}$,wesay that (a, b) is a twin zero of $\mathrm{i}$ if $\mathrm{ab}=0, \mathrm{a} \not \mathrm{i}, \mathrm{b} \not \mathrm{i}$.

Remark 3.2)If $i$ is weakly prime element of $L$ that is not a primeelement then $i$ has twin zero $(a, b)$ for some $a, b \in L$.

Theorem 3.3)Let $\mathrm{i}$ be a weakly prime element of $\mathrm{L}$ and supposethat $(a, b)$ is a twin zero of $i$ for some $a, b \in L$. Then $\mathrm{ai}=\mathrm{bi}=0$.

Proof:- Suppose ai $\neq 0$. Then there exists $c \leq i$ such that ac $\neq$ 0 . Hence $a(b \vee c) \neq 0$. Since $(a, b)$ is a twin zero of $i$ and $a b$ $=0$, we have a $\not \mathrm{i}$ and $\mathrm{b} \not \mathrm{i}$. As a $\not \mathrm{i}$, $\mathrm{i}$ is weakly prime and $0 \neq \mathrm{a}(\mathrm{b} \vee \mathrm{c}) \leq \mathrm{ac} \leq \mathrm{i}$. We must have $\mathrm{b} \leq \mathrm{c} \leq \mathrm{i}$. Hence $\mathrm{b} \leq \mathrm{i}$, $\mathrm{a}$ contradiction. Hence ai $=0$ and similarly it can be shown that $\mathrm{bi}=0$.

Theorem 3.4)Leti be a weakly prime element of $L$. If $i$ is notprime then $i^{2}=0$.

Proof:-Let $(\mathrm{a}, \mathrm{b})$ be twin zero of $\mathrm{i}$. Hence $\mathrm{ab}=0$, where a $\$ \mathrm{i}$ and $\mathrm{b} \not \mathrm{i}$. Assume that $i^{2} \neq 0$. Suppose $i_{1} \cdot i_{2} \neq 0$ for some $i_{1}, i_{2} \leq \mathrm{i}$. Then $\left(\mathrm{a} \vee i_{1}\right)\left(\mathrm{b} \vee i_{2}\right)=i_{1} . i_{2} \neq 0$ (by Theorem 3.3). Since $0 \neq\left(\mathrm{a} \vee i_{1}\right)\left(\mathrm{b} \vee i_{2}\right) \leq \mathrm{i}$ and $\mathrm{i}$ is weakly prime, it follows that $\left(\mathrm{a} \vee i_{1}\right) \leq$ ior $\left(\mathrm{b} \vee i_{2}\right) \leq \mathrm{i}$. Thus $\mathrm{a} \leq \mathrm{i}$ or $\mathrm{b} \leq \mathrm{i}$, which is a contradiction. Therefore $i^{2}=0$.

Theorem 3.5)Leti be a weakly prime element of L. If $i$ is notprime then $i \leq \sqrt{0}$ and $i \sqrt{0}=0$.

Proof:- Suppose $\mathrm{i}$ is not prime. Then by Theorem (3.4), $i^{2}=$ 0 and hence $i \leq \sqrt{0}$. Let $a=\sqrt{0}$. If $a \leq i$, then $a i=0$, by Theorem(3.4). Now assume that a $\$ \mathrm{i}$ and ai $\neq 0$. Hence $\mathrm{ab} \neq$ 0 for some $\mathrm{b} \leq \mathrm{i}$. Let $\mathrm{m}$ be the least positive integer such that $a^{m}=0$. Since $\mathrm{a}\left(a^{m-1} \vee \mathrm{b}\right)=\mathrm{ab} \neq 0$ and $\mathrm{a} \$ \mathrm{i}$, we have, $\left(a^{m-1} \vee \mathrm{b}\right) \leq \mathrm{i}$. Since $0 \neq a^{m-1} \leq \mathrm{i}$ and $\mathrm{i}$ is weakly prime, we conclude that $\mathrm{a} \leq \mathrm{i}$, a contradiction. Thus ai $=0$ for all $\mathrm{a} \leq \sqrt{0}$. Therefore $\mathrm{i} \sqrt{0}=0$.

Theorem 3.6)Let $\mathrm{i}$ be a weakly prime element of $\mathrm{L}$ and $\operatorname{suppose}(a, b)$ is twin zero of $i$. If ar $\leq i$ for some $r \in L$, then ar $=0$.

Proof:- Suppose $0 \neq$ ar $\leq \mathrm{i}$ for some $r \in \mathrm{L}$. Since $(\mathrm{a}, \mathrm{b})$ is twinzero of $\mathrm{i}, \mathrm{ab}=0$ where $\mathrm{a} \not \mathrm{i}$ and $\mathrm{b} \$ \mathrm{i}$. As $\mathrm{i}$ is weakly prime and $0 \neq$ ar $\leq \mathrm{i}$, it follows that $\mathrm{r} \leq \mathrm{i}$. By Theorem (3.3), ai $=$ bi $=0$. Hence $\mathrm{r} \leq \mathrm{i}$ implies ar $=0$, a contradiction. Therefore ar $=0$.

Theorem 3.7) Let $i$ be a weakly prime element of $L$. Supposeab $\leq \mathrm{i}$ for some $\mathrm{a}, \mathrm{b} \in \mathrm{L}$. If $\mathrm{i}$ has twin zero $a_{1}, b_{1}$ for some $a_{1} \leq$ aand $b_{1} \leq \mathrm{b}$ then $\mathrm{ab}=0$.

Proof:- Suppose $\left(a_{1}, b_{1}\right)$ is a twin zero of $\mathrm{i}$ for some $a_{1} \leq \mathrm{a}$ and $b_{1} \leq \mathrm{b}$ and assume that $\mathrm{ab} \neq 0$. Hence $\mathrm{cd} \neq 0$ for some $\mathrm{c}$ $\leq \mathrm{a}$ andd $\leq \mathrm{b}$. Now $0 \neq \mathrm{cd} \leq \mathrm{ab} \leq \mathrm{i}$, where $\mathrm{i}$ is weakly prime. Hence $\mathrm{c} \leq$ ior $\mathrm{d} \leq \mathrm{i}$. Without loss of generality, we may assume that $\mathrm{c} \leq \mathrm{i}$. ByTheorem (3.4), $i^{2}=0$. If $\mathrm{d} \leq \mathrm{i}$ then $\mathrm{c} \leq \mathrm{i}$ implies $\mathrm{cd} \leq i^{2}=0$ andhence $\mathrm{cd}=0$, a contradiction. Therefore $\mathrm{d} \not \mathrm{i}$. Next $\mathrm{ab} \leq \mathrm{i}, \mathrm{d} \leq$ bimplies ad $\leq \mathrm{i}$. Also $a_{1} \not \mathrm{i}$ gives a $\$ \mathrm{i}$. As $\mathrm{i}$ is weakly prime a $\$ \mathrm{i}, \mathrm{d} \$ \mathrm{i}$ and ad $\leq \mathrm{i}$ implies $\mathrm{ad}=0$. Since $\left(a_{1} \vee \mathrm{c}\right) \mathrm{d}=a_{1} \mathrm{~d} \vee \mathrm{cd}=\mathrm{cd} \neq 0$. Now $0 \neq\left(a_{1} \vee\right.$ c) $\mathrm{d}=\mathrm{cd} \leq \mathrm{i}, \mathrm{i}$ is weakly prime, $\mathrm{d} \not \leq \mathrm{i}$ together imply $a_{1} \vee \mathrm{c} \leq$ i. So $a_{1} \leq \mathrm{i}$, a contradiction. Hence $\mathrm{ab}=0$.

The following result is proved by Calliap et.al.[9]

But this result is an outcome of the results proved above whoseproof is different.

Corollary 1) Let $\mathrm{p}$ and $\mathrm{q}$ be weakly prime elements of $\mathrm{L}$ whichare not prime then $\mathrm{pq}=0$.

Proof:- By Theorem (3.5), $\mathrm{p}, \mathrm{q} \leq \sqrt{0}$. Hence $\mathrm{pq} \leq \mathrm{p} \sqrt{0}=0$ (ByTheorem 3.5). Thus pq $=0$.

\section{Triple zeros of weakly $\mathbf{2}$-absorbing elements:}

The concept of a triple zero of a weakly 2-absorbing ideal and free triple zero of weakly 2-absorbing ideal in a commutative ring is defined and studied by A. Badawi Certain Generalized Prime Elements et.al.[20]. The concept of a triple zero of a weakly 2-absorbing primary element is defined and studied by C.S.Manjarekar et.al.[54]. We extend the concept of a triple zero and free triple zero of a 
weakly 2-absorbing element in a compactly generated multiplicative lattices and obtain their properties.

Definition (3.9) Let $i$ be a weakly 2-absorbing element of a multiplicative lattice $\mathrm{L}$ and $\mathrm{a}, \mathrm{b}, \mathrm{c} \in \mathrm{L}$. We say that $(\mathrm{a}, \mathrm{b}, \mathrm{c})$ is a triple zero of $i$ if $a b c=0, a b \$ i, b c \notin i, a c \notin i$.

Definition (3.10) Let $i$ be a weakly 2-absorbing element of a multiplicative lattice $\mathrm{L}$ and suppose $a_{1} a_{2} a_{3} \leq \mathrm{i}$ for some elements $a_{1}, a_{2}, a_{3} \in \mathrm{L}$. We say that $\mathrm{i}$ is a free triple zero with respect to $a_{1} a_{2} a_{3}$ if $(\mathrm{a}, \mathrm{b}, \mathrm{c})$ is not a triple zero of $\mathrm{i}$ for any a $\leq a_{1}$,

$\mathrm{b} \leq a_{2}, \mathrm{c} \leq a_{3}$.

Example 3.11) Let $R=Z 90$. The set $L=\{i \mid i$ is an ideal of $\mathrm{R}\}$ is a compactly generated multiplicative lattice. $\mathrm{L}=\{0,<$ $1>,\langle 2\rangle,\langle 3\rangle,\langle 5\rangle,\langle 6\rangle,\langle 9\rangle,<10\rangle,<15\rangle,<18>,<30$ $>,<45>$ \}. Then $\mathrm{I}=<30>2 \mathrm{~L}$ and $06=<2><3><5\rangle_{-}$I $=\langle 30\rangle$ but $\langle 2\rangle\langle 3\rangle *\langle 30\rangle,\langle 2\rangle\langle 5\rangle *\langle 30\rangle,\langle 3\rangle<5$ $>*<30>=$ I. Hence iis not weakly 2 -absorbing element of $\mathrm{L}$.

Lemma 3.12) Let $i$ be a weakly 2 -absorbing element of $L$ and suppose abd $\leq$ ifor some elements $a, b, d \in$ L such that $(a, b, c)$ is not a triple zero of $i$ for every $c \leq d$. If $a b \notin i$, then ad $\leq \mathrm{i}$ or bd $\leq \mathrm{i}$.

Proof:-Suppose ad $\$ \mathrm{i}$ or $\mathrm{bd} \not \mathrm{i}$. Then $\mathrm{a} d_{1} \not \mathrm{i}$ and $\mathrm{b} d_{2} \not \mathrm{i}$ for some $d_{1}, d_{2} \leq \mathrm{d}$.Since $\left(\mathrm{a}, \mathrm{b}, d_{1}\right)$ is not a triple zero of $\mathrm{i}$ and $\mathrm{ab} d_{1} \leq \mathrm{i}$ and $\mathrm{ab} \not \mathrm{i}, \mathrm{a} d_{1} \Varangle \mathrm{i}$, we have $\mathrm{b} d_{1} \leq \mathrm{i}$. Since $\left(\mathrm{a}, \mathrm{b}, d_{2}\right)$ is not a triple zero of $\mathrm{i}$ and $\mathrm{ab} d_{2} \leq \mathrm{i}$ and $\mathrm{ab} \not \mathrm{i}, \mathrm{b} d_{2} \not \mathrm{i}$, we have $\mathrm{a} d_{2} \leq \mathrm{i}$. Now since $\left(\mathrm{a}, \mathrm{b},\left(d_{1} \vee d_{2}\right)\right)$ is not a triple zero of $\mathrm{i}$ and $\mathrm{ab}\left(d_{1} \vee d_{2}\right) \leq \mathrm{i}$ and ad $\not \mathrm{i}$, we have $\mathrm{a}\left(d_{1} \vee d_{2}\right) \leq \mathrm{i}$ or $\mathrm{b}\left(d_{1} \vee d_{2}\right) \leq \mathrm{i}$. Suppose $\mathrm{a}\left(d_{1} \vee d_{2}\right)=\mathrm{a} d_{1} \vee \mathrm{a} d_{2} \leq \mathrm{i}$. Since $\mathrm{a} d_{2}$ $\leq \mathrm{i}$ and $\mathrm{a} d_{1} \leq \mathrm{i}$, we have a contradiction. Now suppose $\mathrm{b}\left(d_{1}\right.$ $\left.\vee d_{2}\right)=\mathrm{b} d_{1} \vee \mathrm{b} d_{2} \leq \mathrm{i}$. Since $\mathrm{b} d_{1} \leq \mathrm{i}$ and $\mathrm{b} d_{2} \leq \mathrm{i}$, we have a contradiction. Hence ad $\leq \mathrm{i}$ or $\mathrm{bd} \leq \mathrm{i}$.

Corollary 3.13) Let $\mathrm{i}$ be a weakly 2 -absorbing element of $\mathrm{L}$ and suppose $a_{1} a_{2} a_{3} \leq \mathrm{i}$ for some elements $a_{1}, a_{2}, a_{3} \in \mathrm{L}$ such that $i$ is a free triple zero with respect to $a_{1} a_{2} a_{3}$. Then if $\mathrm{a} \leq a_{1}, \mathrm{~b} \leq a_{2}, \mathrm{c} \leq a_{3}$, then $\mathrm{ab} \leq \mathrm{i}$ or $\mathrm{bc} \leq \mathrm{i}$ or $\mathrm{ac} \leq \mathrm{i}$.

Proof:- Since $\mathrm{i}$ is a free triple zero with respect to $a_{1} a_{2} a_{3}$. It follows that $(\mathrm{a}, \mathrm{b}, \mathrm{c})$ is not a triple zero of $\mathrm{i}$ for every $\mathrm{a} \leq a_{1}$, $\mathrm{b} \leq a_{2}, \mathrm{c} \leq a_{3}$. We have abc $\leq a_{1} a_{2} a_{3} \leq \mathrm{i}$. Since $(\mathrm{a}, \mathrm{b}, \mathrm{c})$ is not a triple zero of $i$ we must have either $a b \leq \mathrm{i}$ or $b c \leq i$ or $a c \leq i$, ifabc $=0$. If $a b c \neq 0$ then $0 \neq a b c \leq \mathrm{i}$ implies $a b \leq \mathrm{i}$ or $\mathrm{bc} \leq \mathrm{i}$ or ac $\leq \mathrm{i}$. Since iis weakly 2 -absorbing element of $\mathrm{L}$.

Theorem 3.14)i is weakly 2 -absorbing element of $\mathrm{L}$ and 0 $\neq a_{1} a_{2} a_{3} \leq \mathrm{i}, a_{1}, a_{2}, a_{3} \in \mathrm{L}$ such that $\mathrm{i}$ is a free triple zero with respect to $a_{1} a_{2} a_{3}$. Then $a_{1} a_{2} \leq \mathrm{i}$ or $a_{2} a_{3} \leq \mathrm{i}$ or $a_{1} a_{3} \leq \mathrm{i}$. Suppose $a_{1} a_{2} \not \mathrm{i}$, we claim that $a_{1} a_{3} \leq \mathrm{i}$ or $a_{2} a_{3} \leq \mathrm{i}$. Suppose $a_{1} a_{3} \not \mathrm{i}$ or $a_{2} a_{3} \not \mathrm{i}$. Then there exist $q_{1} \leq a_{1}$ and $q_{2} \leq a_{2}$ such that $q_{1} a_{3} \not \mathrm{i}$ and $q_{2} a_{3} \not \mathrm{i}$. Since $\mathrm{q} q_{2} a_{3} \leq \mathrm{i}$ and $q_{1} a_{3} \not \mathrm{i}$, $q_{2} a_{3} \not \mathrm{i}$, we have $q_{1} q_{2} \leq \mathrm{i}$ by lemma (3.12). Since $a_{1} a_{2} \not$ iwe have $\mathrm{ab} \not \mathrm{i}$ for some $\mathrm{a} \leq a_{1}, \mathrm{~b} \leq a_{2}$. Since $\mathrm{ab} a_{3} \leq \mathrm{i}$ and $\mathrm{ab} \notin \mathrm{i}$, we have $a a_{3} \leq \mathrm{i}$ or $\mathrm{b} a_{3} \leq \mathrm{i}$ by lemma (3.12).

Proof:-Case 1) Suppose a $a_{3} \leq \mathrm{i}$ but $\mathrm{b} a_{3} \notin \mathrm{i}$. Since $q_{1} \mathrm{~b} a_{3} \leq \mathrm{i}$ and $\mathrm{b} a_{3} \not \mathrm{i} q_{1} a_{3} \neq \mathrm{i}$ and we have $q_{1} \mathrm{~b} \leq \mathrm{i}$ by lemma (3.12). Since (a $\left.\vee q_{1}\right) \mathrm{b} a_{3} \leq \mathrm{i} q_{1} a_{3} \sharp \mathrm{i}$ we conclude that (a $\left.\vee q_{1}\right) a_{3} \not$ i. Since $b a_{3} \not \mathrm{i}$ and $\left(\mathrm{a} \vee q_{1}\right) a_{3} \not \mathrm{i}$ we conclude that $\left(\mathrm{a} \vee q_{1}\right) \mathrm{b} \leq \mathrm{i}$ by lemma(3.12). Since $\left(\mathrm{a} \vee q_{1}\right) \mathrm{b}=\mathrm{ab} \vee q_{1} \mathrm{~b}$ $\leq \mathrm{i}$, so $\mathrm{ab} \leq \mathrm{i}$, a contradiction.

Case 2) Suppose $\mathrm{b} a_{3} \leq \mathrm{i}$ but $\mathrm{a} a_{3} \not \mathrm{i}$. Since $\mathrm{a} q_{2} a_{3} \leq \mathrm{i}$ and $\mathrm{a} a_{3} \not \mathrm{i}, q_{2} a_{3} \not \mathrm{i}$ we conclude that $\mathrm{a} q_{2} \leq \mathrm{i}$. Since $\mathrm{a}\left(\mathrm{b} \vee q_{2}\right) a_{3} \leq \mathrm{i}$ and $q_{2} a_{3} \notin \mathrm{i}$ we conclude (b $\left.\vee q_{2}\right) a_{3} \notin \mathrm{i}$. Since $\mathrm{a} a_{3} \notin \mathrm{i}$, $\left(\mathrm{b} \vee q_{2}\right) a_{3} \not \mathrm{i}$, we conclude that $\mathrm{a}\left(\mathrm{b} \vee q_{2}\right) \leq \mathrm{i}$ by lemma (3.12). Since $\mathrm{a}\left(\mathrm{b} \vee q_{2}\right)=\mathrm{ab} \vee \mathrm{a} q_{2} \leq \mathrm{i}$, we have $\mathrm{ab} \leq \mathrm{i}$, a contradiction.

Case 3) Suppose $\mathrm{a} a_{3} \leq \mathrm{i}$ and $\mathrm{b} a_{3} \leq \mathrm{i}$. Since $q_{2} a_{3} \not \mathrm{i}$, we conclude that $\left(\mathrm{b} \vee q_{2}\right) a_{3} \notin \mathrm{i}$. Since $q_{1}\left(\mathrm{~b} \vee q_{2}\right) a_{3} \leq \mathrm{i}$ and $q_{1} a_{3} \notin \mathrm{i}$, (b $\left.\vee q_{2}\right) a_{3} \not$ i so $q_{1}\left(\mathrm{~b} \vee q_{2}\right)=q_{1} \mathrm{~b} \vee q_{1} q_{2} \leq \mathrm{i}$ by lemma (3.12). Since $\left(q_{1} \mathrm{~b} \vee q_{1} q_{2}\right) \leq \mathrm{i}$ we conclude $\mathrm{b} q_{1} \leq \mathrm{i}$. As $q_{1} a_{3} \not \mathrm{i}$, ( $\mathrm{a} \vee$ $\left.q_{1}\right) a_{3} \not$ i. Since $\left(\mathrm{a} \vee q_{1}\right) q_{2} a_{3} \not \mathrm{i}$ and $q_{2} a_{3} \not$ i. $\left(\mathrm{a} \vee q_{1}\right) a_{3} \not \mathrm{i}$ we have $\left(\mathrm{a} \vee q_{1}\right) q_{2}=\mathrm{a} q_{2} \vee q_{1} q_{2} \leq \mathrm{i} \quad$ so $\mathrm{a} q_{2} \leq \mathrm{i}$. Now since (a $\left.\vee q_{1}\right)\left(\mathrm{b} \vee q_{2}\right) a_{3} \leq \mathrm{i}$ and $\left(\mathrm{a} \vee q_{1}\right) a_{3} \not \mathrm{i}$ and $\left(\mathrm{b} \vee q_{2}\right) a_{3} \not$ iwe have $\left(\mathrm{a} \vee q_{1}\right)\left(\mathrm{b} \vee q_{2}\right)=\mathrm{ab} \vee \mathrm{a} q_{2} \vee \mathrm{b} q_{1} \vee q_{1} q_{2} \leq \mathrm{i}$. By lemma (3.12) we concludethat $\mathrm{ab} \leq \mathrm{i}$, a contradiction. Hence $a_{1} a_{3} \leq$ i or $a_{2} a_{3} \leq \mathrm{i}$.

\section{REFERENCES}

[1] D.D. Anderson, Abstract commutative ideal theory without chain condition, Algebra Universalis,6,(1976), 131-145.

[2] R.P. Dilworth , Abstract Commutative Ideal theory, Pacific. J. Math.,12,(1962)481-498.

[3] F. Alarcon, D.D. Anderson, C. Jayaram, Some results on abstract commutative ideal theory, Periodica Mathemetica Hungerica, Vol 30 (1), (1995),pp.1-26.

[4] N. K. Thakre, C.S. Manjarekar and S. Maida, Abstract spectral theory II,Minimal characters and minimal spectrum of multiplicative lattices, Acta Sci.Math., 52 (1988) 53-67.

[5] Tessema, Belayneh, Venkateshwarlu, Certain Generalized Prime Ideals InBoolean like Semirings,International J. of Algebra, Hikari Ltd., Vol.8,(2014)No.14,663-669.

[6] U. Tekir,E. Yetkin,A. Badawi on 2 absorbing primary ideals in commutative rings, Bull korean Math. Soc.,51(2014), No.4,1163-1173.

[7] U. Tekir,E. Yetkin,C. Jayaram 2-absorbing and weakly 2-absorbing elements in multiplicatiove lattices, Communications in Algebra, 42,(2014),23382353.

[8] U. Tekir,E. Yetkin,F. Callialp On 2-absorbing primary and weakly 2-absorbingelements in multiplicative lattices,Italian Journal of Pure and Applied Mathematics, 34-2005, 263-276.

[9] C. Jayaram, U. Tekir, F.Callialp weakly prime elements in Multiplicative Lattices, Communications In Algebra 40, 2825-2840, 2012. 